\title{
Ultra-wideband dual-polarization silicon nitride power splitter based on modal engineered slot waveguides
}

\author{
DaVid GonzÁlez-Andrade, ${ }^{1, *}$ Sylvain Guerber, ${ }^{2,3}$ Elena DurÁN- \\ Valdeiglesias, ${ }^{2}$ Diego Pérez-Galacho, ${ }^{2,4}$ XaVier le RouX, ${ }^{2}$ Nathalie \\ Vulliet, ${ }^{3}$ SÉbastien Cremer, ${ }^{3}$ Stephane Monfray, ${ }^{3}$ Eric Cassan, ${ }^{2}$ Delphine \\ Marris-Morini, ${ }^{2}$ Frédéric Boeuf, ${ }^{3}$ Pavel Cheben, ${ }^{5}$ LaUrent Vivien, ${ }^{2}$ Aitor \\ V. Velasco, ${ }^{1}$ and Carlos Alonso-Ramos ${ }^{2}$
}

\author{
${ }^{1}$ Instituto de Óptica Daza de Valdés, Consejo Superior de Investigaciones Científicas (CSIC), Madrid 28006, Spain \\ ${ }^{2}$ Centre de Nanosciences et de Nanotechnologies, CNRS, Université Paris-Sud, Université Paris-Saclay, 91120 Palaiseau, France \\ ${ }^{3}$ STMicroelectronics SAS-850 rue Jean Monnet-38920 Crolles, France \\ 4 ITEAM Research Institute, Universitat Politécnica de Valencia, Camino de Vera $s / n, 46022$ Valencia, Spain \\ ${ }^{5}$ National Research Council Canada, 1200 Montreal Road, Bldg. M50, Ottawa K1A OR6, Canada \\ *Corresponding author: david.gonzalez@csic.es
}

Received XX Month XXXX; revised XX Month, XXXX; accepted XX Month XXXX; posted XX Month XXXX (Doc. ID XXXXX); published XX Month XXXX

\begin{abstract}
Silicon nitride (SiN) waveguides provide a substantially lower index contrast, thermo-optic coefficient and reduced birefringence compared to silicon-on-insulator waveguides. These properties make SiN a prominent candidate for implementation of ultra-wideband dualpolarization photonics circuits with a great potential for datacom applications. State-of-the-art SiN power splitters are still hampered in terms of either bandwidth or singlepolarization operation. Here, we propose to overcome these limitations by exploiting modal and waveguide symmetry engineering in a single-mode slot waveguide. This topology prevents mode-beating, while granting symmetric power splitting for both polarizations. Experimental characterization of the fabricated device shows low loss $(<0.62 \mathrm{~dB})$ and imbalance $(<0.6 \mathrm{~dB})$ within an unprecedented bandwidth of $420 \mathrm{~nm}(1.26 \mu \mathrm{m}-1.68$ $\boldsymbol{\mu m})$. (C) 2019 Optical Society of America
\end{abstract}

http://dx.doi.org/10.1364/OL.99.099999

In the present era of information, internet traffic is growing relentlessly driven primarily by emerging cloud services, Internet of Things (IoT) architectures and 5G communications. This massive traffic demand caused by an increasing number of connected devices has long exceeded the Zettabyte per year and is expected to keep rising in the forthcoming years, especially in datacenters [1-3]. Silicon photonics ( $\mathrm{SiP}$ ) has proven to be a promising solution to tackle this challenge, owing to its low energy consumption, compatibility with complementary metal-oxide-semiconductor (CMOS) foundries and high integration density [4].

The silicon-on-insulator (SOI) platform has become mainstream for the design and implementation of SiP circuits. However, the high refractive index contrast between the silicon waveguide core and the silicon oxide cladding $(\Delta n \approx 2)$ poses some important challenges in terms of tight fabrication tolerances and strong polarization dependence. In order to overcome these constraints, silicon nitride ( $\mathrm{SiN}$ ) has been proposed as a promising alternative $(\Delta n \approx 0.5)$. SiN benefits include enhanced resilience to fabrication deviations, wider transparency window reaching the visible range, lower scattering losses and reduced birefringence between transverse electric (TE) and transverse magnetic (TM) polarizations [5], enabling the implementation of dual-polarization devices while relative compact footprints are maintained [6,7]. Many applications based on the SiN platform have been demonstrated, including spectroscopy, datacom and quantum photonics, among others [8-10]. In the majority of these applications, splitting or combining the light beam inside the chip is of fundamental importance with optical power splitters being a key building block.

Although many implementations of beam splitters have been experimentally demonstrated in the SOI platform, most of them suffer either from single-polarization operation, narrow bandwidths or stringent fabrication tolerances, and would greatly benefit from the lower refractive index contrast of SiN. For example, directional couplers (DCs) and multimode interference (MMI) devices rely on a mode-beating effect limiting their operational bandwidths $[11,12]$. Subwavelength grating (SWG) waveguides have been successfully applied to Si-based DCs and MMIs to 
improve their bandwidths; however, this remarkable performance is only achieved for a single polarization $[13,14]$. On the other hand, symmetric Y-junctions are hampered by the limited resolution of fabrication processes, leading to comparatively high insertion losses. Fabrication constraints have been alleviated by means of single-polarization adiabatic splitters [15] and alternative designs of Y-junctions [16]. Polarization-agnostic beam splitters have also been implemented in SOI based on bent DCs, adiabatic couplers and tapered waveguides [17-19], with limited experimental bandwidth of less than $100 \mathrm{~nm}$. Among these alternatives, we recently proposed an ultra-wideband nanophotonic beam splitter with an experimental bandwidth of $390 \mathrm{~nm}$ for both TE and TM polarizations [20].

Conversely, in the SiN platform just a few types of power splitters have been reported. Table 1 summarizes the performance of both simulated and experimental SiN power splitters [10,21-27]. Proposed solutions include DCs [10], MMIs [21], asymmetric DCs (ADCs) [22], wavelength-stabilized DCs (WSDC) [23], ring resonators (RRs) [24], adiabatic tapers [25] and MMIs [26,27]. It should be noted that only the adiabatic-taper-based solution shows a simulated ultra-wideband performance over a $300 \mathrm{~nm}$ wavelength range for both polarizations [25]. Nevertheless, no experimental results based on this architecture have been reported yet. An ultra-wideband dual-polarization power splitter is still sought-after for the implementation of next generation photonic integrated circuits based on the SiN platform.

Table 1. Simulated (*) and experimental insertion loss (IL), imbalance (IB) and bandwidth (BW) of state-of-the-art silicon nitride power splitters. Worst performance was considered in dual-polarization splitters.

\begin{tabular}{lcccc}
\hline Ref. & IL $\mathbf{( d B})$ & IB $(\mathbf{d B})$ & BW $(\mathbf{n m})$ & TE/TM \\
\hline$[10]$ & $<0.06$ & $<0.01$ & - & TE \\
\hline$[21]^{*}$ & $<0.37$ & - & 70 & TE and TM \\
\hline$[22]^{*}$ & $<0.18$ & $<0.30$ & 100 & TE and TM \\
\hline$[23]$ & - & $<1.05$ & 100 & TE \\
\hline$[24]$ & $<0.90$ & $<0.40$ & - & TE \\
\hline$[25]^{*}$ & $<1.00$ & $<1.76$ & 300 & TE and TM \\
\hline$[26]$ & $<0.10$ & - & - & TE \\
\hline$[27]$ & $<0.32$ & $<0.87$ & - & TE \\
\hline This work & $<0.62$ & $<0.6$ & 420 & TE and TM \\
\hline
\end{tabular}

In this Letter, we propose to leverage our modal and symmetry engineering design strategy [20] for the SiN platform. Following this approach, our optical power splitter precludes the excitation and beating of higher-order modes due to a single-mode slot design, whereas the two symmetric tapers at the output ensure an equal power splitting. Moving from SOI to SiN implies a substantial decrease in the refractive index contrast. In addition, the rail widths and their separation have to be increased to satisfy the minimum feature size during the fabrication on the SiN platform. Hence, the splitter had to be thoroughly redesigned for the SiN technology. The proposed device was fabricated in a 600-nm-thick silicon nitride layer. We experimentally demonstrate insertion loss lower than $0.62 \mathrm{~dB}$ and an imbalance below $0.6 \mathrm{~dB}$ over an unprecedented bandwidth of $420 \mathrm{~nm}$ for both TE and TM polarizations.

Figure 1 shows the device schematic of the proposed $1 \times 2$ silicon nitride power splitter, which comprises a strip-to-slot mode converter (length $L_{A}+L_{B}$ ), a slot waveguide (length $L_{S}$ ) and two

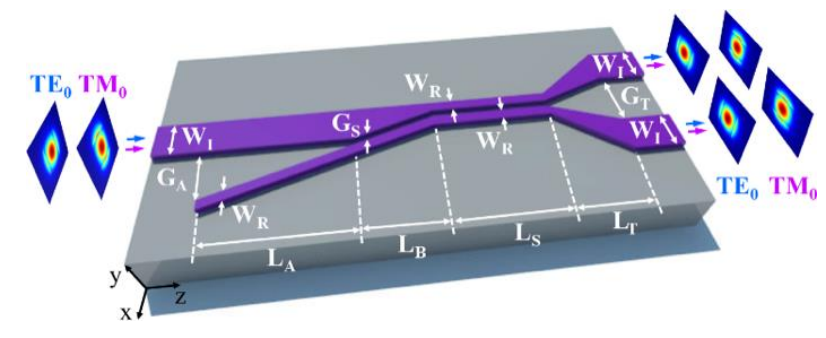

Fig. 1. Schematic of the proposed silicon nitride power splitter based on a single-mode slot waveguide. TE-polarized light (labeled in blue) and TM-polarized light (magenta) are equally split by the two symmetric output tapers in a wavelength agnostic manner.

symmetric output tapers (length $L_{T}$ ). Our design strategy relies on modal engineering in the slot waveguide to ensure single-mode condition within the entire wavelength range, which prevents mode-beating between the fundamental TE or TM mode and other higher-order modes. At the same time, symmetry of the slot and the output splitting structure achieves an equal power splitting and improved resilience to fabrication errors. Furthermore, the generation of a tip in the splitting transition during the fabrication process (as occurs in symmetric Y-junctions) is circumvented due to the inherent gap of slot waveguides.

For the design, we considered a 600-nm-thick silicon nitride layer surrounded by a silicon dioxide $\left(\mathrm{SiO}_{2}\right)$ upper and lower layers. The slot waveguide of the splitter consists of two narrow waveguides or rails (width $W_{R}$ ) separated by a central gap (width $G_{S}$ ). In order to achieve an ultra-wideband performance, this structure needs to be single mode within the entire wavelength range considered for simulation $(1.2-1.7 \mu \mathrm{m})$. Consequently, the design of the slot waveguide is first carried out by judiciously selecting the width values of rails and slot that fulfil the single-mode condition. We studied the effective index variation with the wavelength using a Finite Difference Eigenmode (FDE) solver [28]. Figure 2(a) shows the effective index of fundamental and first-order modes in the slot waveguide for both TE and TM polarizations, for

a)

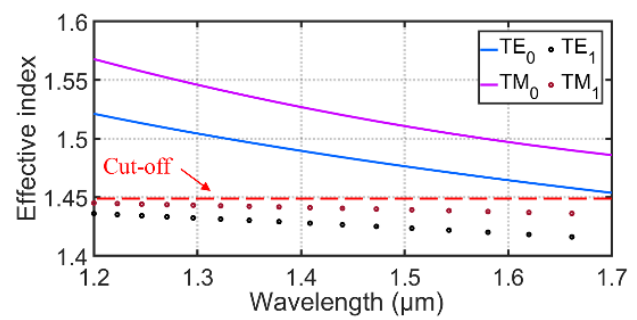

b)

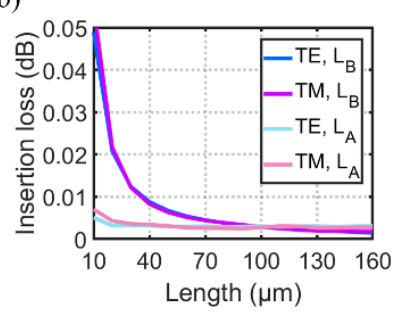

c)

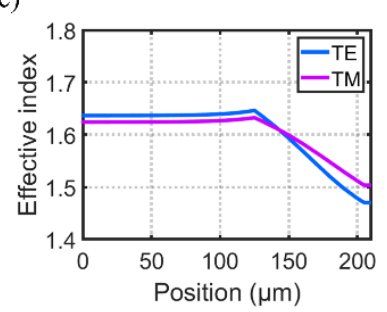

Fig. 2. (a) Calculated effective index of fundamental and first-order TE and TM modes in a slot waveguide with $W_{R}=G_{S}=200 \mathrm{~nm}$. (b) Insertion loss of the strip-to-slot mode converter as a function of $L_{A}$ and $L_{B}$. (c) Effective index of the fundamental TE and TM modes along the strip-to-slot mode converter. 

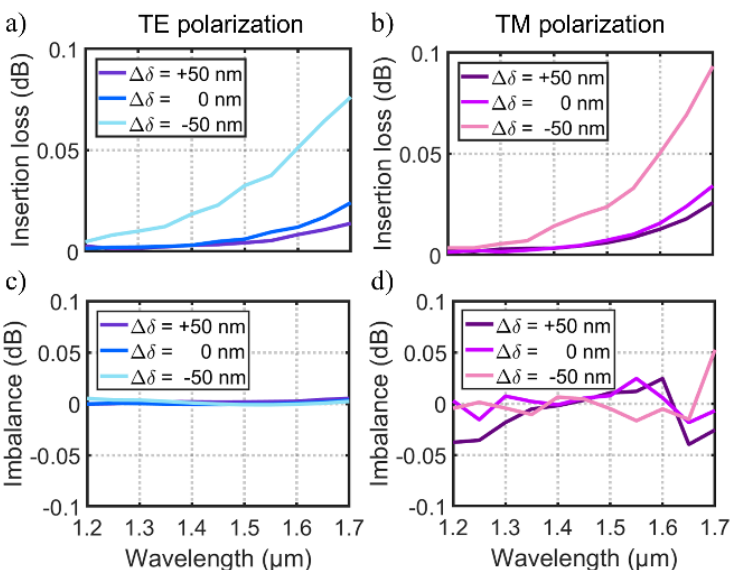

Fig. 3. Simulated insertion loss of the proposed SiN power splitter for (a) TE and (b) TM polarizations. Simulated imbalance for (c) TE and (d) TM polarizations. Tolerances to fabrication errors of $\Delta \delta= \pm 50 \mathrm{~nm}$ are also represented for both insertion loss and imbalance.

$W_{R}=G_{S}=200 \mathrm{~nm}$. For these design parameters, first-order TE and TM modes are under cut-off condition for the wavelength range $1.2 \mu \mathrm{m}-1.7 \mu \mathrm{m}$, yielding single-mode slot waveguide. Next, the input strip waveguide with a single-mode width of $W_{I}=700 \mathrm{~nm}$ is transformed into the slot waveguide via an adiabatic strip-to-slot mode converter. We set an initial gap $G_{A}$ of $1 \mu \mathrm{m}$. We studied the insertion loss as a function of the lengths $L_{A}$ and $L_{B}$ separately via 3D bidirectional eigenmode expansion (EME) solver [28]. Lengths of $L_{A}=120 \mu \mathrm{m}$ and $L_{B}=80 \mu \mathrm{m}$ were chosen to ensure an adiabatic transition. This is a very conservative selection of the taper length, considering the loss calculations shown in Fig. 2(b). As shown in Fig. 2(c), the effective index is adiabatically modified along the transition. Nevertheless, fabrication imperfections in the tapers may result in unwanted radiation that might be re-coupled in the output sections. To avoid this, we chose a slot waveguide length $\left(L_{S}\right)$ of $60 \mu \mathrm{m}$ to filter out residual light radiated in the taper. Finally, the output section is designed to ensure equal power splitting. For this purpose, both rails of the slot waveguide are gradually transformed into strip waveguides by means of symmetric tapers. Negligible losses were found for both polarizations for a taper length of $L_{T}=60 \mu \mathrm{m}$ and a separation of $G_{T}=1.5 \mu \mathrm{m}$ between output strip waveguides.

Finally, our device was also simulated by 3D EME (see Fig. 3). An insertion loss lower than $0.04 \mathrm{~dB}$ is obtained for both polarizations over an unprecedented bandwidth of $500 \mathrm{~nm}(1.2-1.7 \mu \mathrm{m})$. Imbalance values for TE and TM polarizations are also negligible (lower than $\pm 0.03 \mathrm{~dB}$ ) for the same wavelength range. Tolerance to fabrication errors was studied for etching errors of $\Delta \delta$. Notice that a waveguide width increase also results in a reduced slot, and vice versa. For over-etching errors, i.e. $\Delta \delta=-50 \mathrm{~nm}$, insertion losses increase to $0.01 \mathrm{~dB}$ at longer wavelengths because both fundamental TE and TM modes approach the cut-off condition. In this case, the worst imbalance value obtained is only $\pm 0.03 \mathrm{~dB}$ for both polarizations within the entire simulated wavelength range. On the other hand, when an under-etching error of $\Delta \delta=+50 \mathrm{~nm}$ is introduced, insertion loss is further reduced to $0.03 \mathrm{~dB}$ and imbalance is lower than $\pm 0.05 \mathrm{~dB}$. These results confirm that the outstanding high performance of our proposed power splitter is preserved even for fabrication errors as large as $\pm 50 \mathrm{~nm}$. The fabrication of the splitter was performed in ST Crolles (France) based on their $300 \mathrm{~mm}$ silicon photonics R\&D platform [29]. The 600 -nm-thick silicon nitride layer was deposited onto a 1.4- $\mu \mathrm{m}$ thick $\mathrm{SiO}_{2}$ layer using low-temperature plasma-enhanced chemical vapour deposition (PECVD). The pattern was defined and transferred via $248 \mathrm{~nm}$ deep-ultraviolet (deep-UV) photolithography and a dry etching process, respectively. Scanning electron microscope (SEM) images of the fabricated splitter were taken (see Fig. 4(a)) and a 1.5- $\mu$ m-thick $\mathrm{SiO}_{2}$ cladding layer was deposited afterwards.

To experimentally validate our power splitter concept, we used Mach-Zehnder interferometer (MZI) test structure with an arm length imbalance of $50 \mu \mathrm{m}$, ensuring a negligible difference in propagation loss between the two arms. Three tunable lasers were connected using a Yenista CT400 to sweep the wavelength range from $1.26 \mu \mathrm{m}$ to $1.68 \mu \mathrm{m}$, exceeding all the telecom bands combined $(\mathrm{O}, \mathrm{E}, \mathrm{S}, \mathrm{C}, \mathrm{L}$ and $\mathrm{U})$. Light was coupled in and out of the chip via fiberchip grating couplers and cleaved single-mode fibers (SMF-28). The same grating couplers were used at the input and output ports of the MZI and the measurement of the whole spectra was carried out by adjusting the tilt angle of the fibers. Figures 4(b) and 4(c) show the measured transmittance spectra of the MZI compared with a reference waveguide for TE and TM polarizations, respectively. The transmission spectra are normalized by the response of the fiberchip grating coupler. High ratio following the procedure in [13], as is shown in Fig. 4(d). The splitter exhibits a measured imbalance of less than $0.58 \mathrm{~dB}$ and 0.6 extinction ratios exceeding $23.5 \mathrm{~dB}$ for TE and $23.3 \mathrm{~dB}$ for TM are observed within a bandwidth of $420 \mathrm{~nm}$.
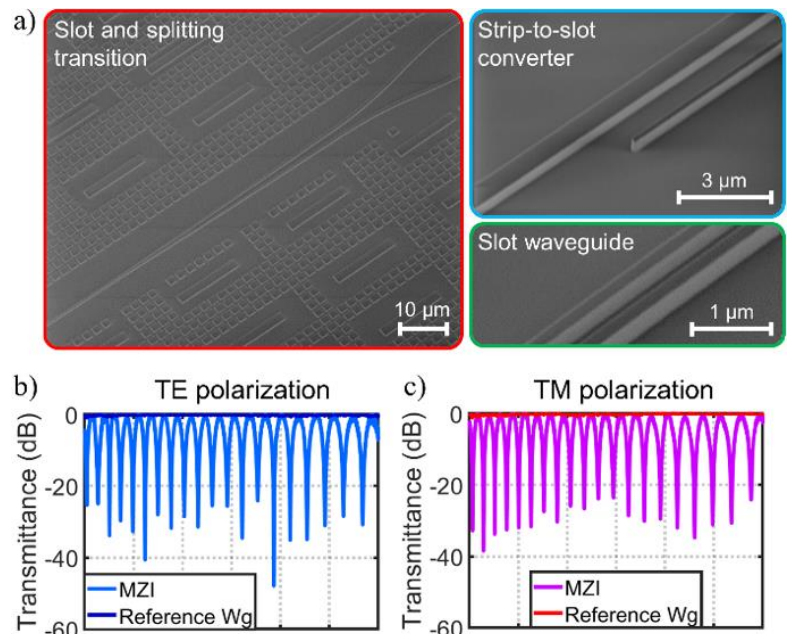

d)
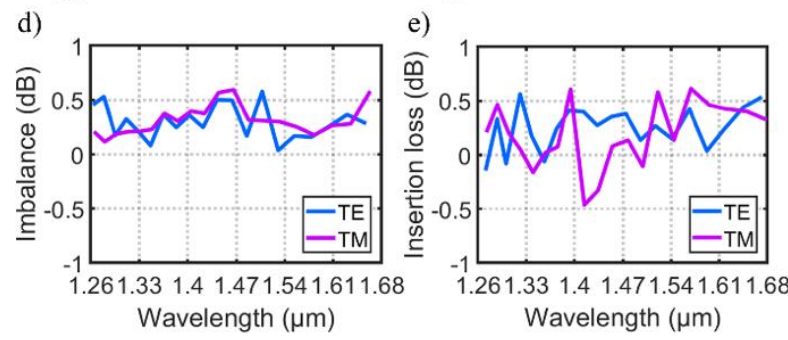

Fig. 4. Scanning electron microscope image (a) of the fabricated power splitter. Measured transmittance spectra of the Mach-Zehnder interferometer for (b) TE and (c) TM polarizations over the $1.26 \mu \mathrm{m}-$ $1.68 \mu \mathrm{m}$ wavelength range. (d) Imbalance and (e) insertion loss calculated from MZI measurements. 
The power imbalance between the output waveguides was extracted from the extinction $\mathrm{dB}$ for TE and TM polarizations, respectively, over the entire measured bandwidth. The insertion loss (IL) is defined as the loss that the signal suffers when it passes through the splitter. We estimate IL from the difference between MZI transmission and a reference waveguide with similar length, without normalization, and taking into account that the MZI comprises two splitters. We found that insertion losses are below $0.62 \mathrm{~dB}$ and $0.57 \mathrm{~dB}$ for TE and TM polarizations, respectively, within the measured wavelength range (Fig. 4(e)). Fine adjustment of the geometry to further compensate fabrication deviations may reduce the imbalance and insertion loss below $0.2 \mathrm{~dB}$, approaching the simulated values. Nevertheless, compared to other SiN power splitters (see Table 1), our proposed device shows an excellent performance within a bandwidth in excess of $420 \mathrm{~nm}$, which makes it one of the most broadband $\mathrm{SiN}$ power splitters ever reported for single- or dual-polarization operation.

In conclusion, we have proposed and experimentally demonstrated a silicon nitride power splitter with ultra-wideband dual-polarization operation. The splitter is based on a modalengineered slot waveguide to fulfill single-mode condition over the entire operating wavelength range and, thus, mitigating any modebeating effects. The splitting transition (slot-to-strip) is designed maintaining the structural symmetry by means of two trapezoidal tapers that ensure an equal power splitting at the outputs. 3D EME simulations show a high-performance over a $500 \mathrm{~nm}$ bandwidth even in the presence of fabrication errors as large as $\pm 50 \mathrm{~nm}$. Fabrication of the proposed power splitter was carried out using a single-etch step process using $248 \mathrm{~nm}$ deep-UV photolithography in a $300 \mathrm{~mm}$ SiN platform, compatible with large-volume industry fabrication. The measurements show low insertion losses of $<0.62$ $\mathrm{dB}$ and low imbalance of $<0.6 \mathrm{~dB}$ for both TE and TM polarizations over an ultra-broad bandwidth exceeding $420 \mathrm{~nm}(1.26 \mu \mathrm{m}-1.68$ $\mu \mathrm{m})$. This is more than a four-fold improvement in bandwidth compared to state-of-the-art silicon nitride power splitters. Furthermore, the length of the device can be reduced by using logarithmic tapers in the strip-to-slot transition [30]. We believe that the proposed power splitter opens new venues as a key building block for broadband and dual-polarization silicon nitride integrated circuits with promising prospects for sensing, spectroscopy and datacom applications.

Funding. Spanish Ministry of Science, Innovation and Universities (MICINN) (TEC2015-71127-C2-1-R with FPI scholarship BES2016-077798, IJCI-2016-30484, RTI2018-097957-B-C33); Community of Madrid - FEDER funds (S2018/NMT-4326); Horizon 2020 research and innovation program (Marie Sklodowska-Curie 734331); H2020 European Research Council (ERC POPSTAR 647342); European Commission (H2020-ICT-26127-2017 COSMICC 688516); French Industry Ministry (Nano2022 project under IPCEI program); Agence Nationale de la Recherche (ANRMIRSPEC-17-CE09-0041).

Disclosures. The authors declare no conflicts of interest.

\section{References}

1. P. J. Winzer, Bell Labs Tech. J. 19, 22 (2014).

2. D. J. Richardson, J. M. Fini, and L. E. Nelson, Nat. Photonics 7, 354 (2013).

3. G. Astfalk, Appl. Phys. A. 95, 933 (2009).
4. P. Dong, K. W. Kim, A. Melikyan, and Y. Baeyens, in IEEE International Electron Devices Meeting (IEDM) 2018 (IEEE, 2018), p. 23.4.1.

5. R. Baets, A. Z. Subramanian, S. Clemmen, B. Kuyken, P. Bienstman, N. Le Thomas, G. Roelkens, D. Van Thourhout, P. Helin, and S. Severi, in Optical Fiber Communication Conference 2016 (Optical Society of America, 2016), p. Th3J.1.

6. E. Durán-Valdeiglesias, S. Guerber, D. Oser, X. Le Roux, D. Benedikovic, D. Pérez-Galacho, N. Vulliet, S. Cremer, S. Monfray, E. Cassan, D. MarrisMorini, C. Baudot, F. Boeuf, L Vivien, and C. Alonso-Ramos, Opt. Lett. 44, 4578 (2019).

7. J. C. Mikkelsen, A. Bois, T. Lordello, D. Mahgerefteh, S. Menezo, and J. K. S. Poon, Opt. Express 26, 30076 (2018).

8. X. Nie, E. Ryckeboer, G. Roelkens, and R. Baets, Opt. Express 25, A409 (2017).

9. P. Dong, IEEE J. Sel. Top. Quantum Electron. 22, 370 (2016).

10. M. Poot, C. Schuck, X. S. Ma, X. Guo, and H. X. Tang, Opt. Express 24, 6843 (2016).

11. H. Yamada, T. Chu, S. Ishida, and Y. Arakawa, IEEE Photonics Technol. Lett. 17,585 (2005).

12. L. B. Soldano and E. C. M. Pennings, J. Lightw. Technol. 13, 615 (1995).

13. Y. Wang, Z. Lu, M. Ma, H.Yun, F. Zhang, N. A. Jaeger, and L. Chrostowski, IEEE Photon. J. 8, 7101408 (2016).

14. R. Halir, P. Cheben, J. M. Luque-González, J D. Sarmiento-Merenguel, J. H. Schmid, G. Wangüemert-Pérez, D.-X. Xu, S. Wang, A. Ortega-Moñux, and Í. Molina-Fernández, Laser Photon. Rev. 10, 1039 (2016).

15. J. Xing, K. Xiong, H. Xu, Z. Li, X. Xiao, J.Yu, and Y. Yu, Opt. Lett. 38, 2221 (2013).

16. Y. Zhang, S. Yang, A. E. J. Lim, G. Q. Lo, C. Galland, T. Baehr-Jones, M. Hochberg, Opt. Express 21, 1310 (2013).

17.X. Chen, W. Liu, Y. Zhang, and Y. Shi, Opt. Lett. 42, 3738 (2017).

18. L. Xu, Y. Wang, D. Patel, E El-Fiky, Z. Xing, R. Li, and D. V. Plant, in Conference on Lasers and Electro-Optics (CLEO) 2017 (IEEE, 2017), p. SF1I.5.

19. Y. Wang, S. Gao, K. Wang, and E. Skafidas, Opt. Lett. 41, 2053 (2016).

20. D. González-Andrade, C. Lafforgue, E. Durán-Valdeiglesias, X. Le Roux, M. Berciano, E. Cassan, D. Marris-Morini, A. V. Velasco, P. Cheben, L. Vivien, and C. Alonso-Ramos, Sci. Rep. 9, 3604 (2019).

21. J. Mu, P. T. Lin, L. Zhan, X. Duan, L. C. Kimerling, and A. M. Agarwal, in Laser Science 2014 (Optical Society of America, 2014), p. JW3A.26.

22. B. Sharma, K. Kishor, S. Sharma, and R. Makkar, Fiber Integrated Opt. 38, 247 (2019).

23. S. Guerber, C. Alonso-Ramos, D. Benedikovic, D. Pérez-Galacho, X. Le Roux, N. Vulliet, S. Crémer, L. Babaud, J. Planchot, D. Benoit, P. Chatraine, F. Leverd, D. Ristoiu, P. Grosse, D. Marris-Morini, L. Vivien, C. Baudot, and F. Boeuf, in Proc. SPIE 10686, Silicon Photonics: From Fundamental Research to Manufacturing 2018, p. 106860W.

24.D. T. Spencer, D. Dai, Y. Tang, M. J. Heck, and J. E. Bowers, IEEE Photon. Technol. Lett. 25, 36 (2012).

25.Z. Yuan and K. Wang, in Asia Communications and Photonics (ACP) 2018 (IEEE, 2018), p. 1.

26. C. V. Poulton, M. J. Byrd, M. Raval, Z. Su, N. Li, E. Timurdogan, D. Coolbaugh, D. Vermeulen, and M. R. Watts, Opt. Lett. 42, 21 (2017).

27. S. Romero-García, F. Merget, F. Zhong, H. Finkelstein, and J. Witzens, Opt. Express 21, 14036 (2013).

28. Lumerical Solutions, Inc., MODE Solutions (2019), http://lumerical .com.

29. S. Guerber, C. Alonso-Ramos, D. Benedikovic, E. Durán-Valdeiglesias, X. Le Roux, N. Vulliet, E. Cassan, D. Marris-Morini, C. Baudot, F. Boeuf, and L. Vivien, IEEE Photon. Technol. Lett. 30, 1679 (2018).

30. R. Palmer, L. Alloatti, D. Korn, W. Heni, P. C. Schindler, J. Bolten, M. Kari, M. Waldow, T. Wahlbrink, W. Freude, C. Koos, and J. Leuthold, IEEE Photonics J. 5, 2200409 (2013). 


\section{Full references}

1. P. J. Winzer, "Spatial multiplexing in fiber optics: the 10x scaling of metro/core capacities," Bell Labs Tech. J. 19, 22-30 (2014).

2. D. J. Richardson, J. M. Fini, and L. E. Nelson, "Space-division multiplexing in optical fibres," Nat. Photonics 7, 354-362 (2013).

3. G. Astfalk, "Why optical data communications and why now?," Appl. Phys. A. 95, 933-940 (2009).

4. P. Dong, K. W. Kim, A. Melikyan, and Y. Baeyens, "Silicon photonics: a scaling technology for communications and interconnects," in IEEE International Electron Devices Meeting (IEDM) 2018 (IEEE, 2018), p. 23.4.123.4.4.

5. R. Baets, A. Z. Subramanian, S. Clemmen, B. Kuyken, P. Bienstman, N. Le Thomas, G. Roelkens, D. Van Thourhout, P. Helin, and S. Severi, "Silicon photonics: silicon nitride versus silicon-on-insulator," in Optical Fiber Communication Conference 2016 (Optical Society of America, 2016), p. Th3J.1.

6. E. Durán-Valdeiglesias, S. Guerber, D. Oser, X. Le Roux, D. Benedikovic, D. Pérez-Galacho, N. Vulliet, S. Cremer, S. Monfray, E. Cassan, D. MarrisMorini, C. Baudot, F. Boeuf, L Vivien, and C. Alonso-Ramos, "Dualpolarization silicon nitride Bragg filters with low thermal sensitivity," Opt. Lett. 44, 4578-4581 (2019).

7. J. C. Mikkelsen, A. Bois, T. Lordello, D. Mahgerefteh, S. Menezo, and J. K. S. Poon, "Polarization-insensitive silicon nitride Mach-Zehnder lattice wavelength demultiplexers for CWDM in the O-band," Opt. Express 26, 30076-30084 (2018).

8. X. Nie, E. Ryckeboer, G. Roelkens, and R. Baets, "CMOS-compatible broadband co-propagative stationary Fourier transform spectrometer integrated on a silicon nitride photonics platform," Opt. Express 25, A409A418 (2017).

9. P. Dong, "Silicon photonic integrated circuits for wavelength-division multiplexing applications," IEEE J. Sel. Top. Quantum Electron. 22, 370-378 (2016).

10. M. Poot, C. Schuck, X. S. Ma, X. Guo, and H. X. Tang, "Design and characterization of integrated components for SiN photonic quantum circuits," Opt. Express 24, 6843-6860 (2016).

11. H. Yamada, T. Chu, S. Ishida, and Y. Arakawa, "Optical directional coupler based on Si-wire waveguides," IEEE Photonics Technol. Lett. 17, 585-587 (2005).

12. L. B. Soldano and E. C. M. Pennings, "Optical multi-mode interference devices based on self-imaging: principles and applications," J. Lightw. Technol. 13, 615-627 (1995).

13. Y. Wang, Z. Lu, M. Ma, H.Yun, F. Zhang, N. A. Jaeger, and L. Chrostowski, "Compact broadband directional couplers using subwavelength gratings," IEEE Photon. J. 8, 7101408 (2016).

14. R. Halir, P. Cheben, J. M. Luque-González, J D. Sarmiento-Merenguel, J. H. Schmid, G. Wangüemert-Pérez, D.-X. Xu, S. Wang, A. Ortega-Moñux, and Í. Molina-Fernández, "Ultra-broadband nanophotonic beamsplitter using an anisotropic sub-wavelength metamaterial," Laser Photon. Rev. 10, 10391046 (2016).

15. J. Xing, K. Xiong, H. Xu, Z. Li, X. Xiao, J.Yu, and Y. Yu, "Silicon-on-insulatorbased adiabatic splitter with simultaneous tapering of velocity and coupling," Opt. Lett. 38, 2221-2223 (2013).

16. Y. Zhang, S. Yang, A. E. J. Lim, G. Q. Lo, C. Galland, T. Baehr-Jones, M. Hochberg, "A compact and low loss Y-junction for submicron silicon waveguide," Opt. Express 21, 1310-1316 (2013).

17. X. Chen, W. Liu, Y. Zhang, and Y. Shi, "Polarization-insensitive broadband 2 $\times 23 \mathrm{~dB}$ power splitter based on silicon-bent directional couplers," Opt. Lett. 42, 3738-3740 (2017).

18. L. Xu, Y. Wang, D. Patel, E El-Fiky, Z. Xing, R. Li, and D. V. Plant, "Polarization independent adiabatic 3-dB coupler for silicon-on-insulator," in Conference on Lasers and Electro-Optics (CLEO) 2017 (IEEE, 2017), p. SF11.5.

19. Y. Wang, S. Gao, K. Wang, and E. Skafidas, "Ultra-broadband and low-loss $3 \mathrm{~dB}$ optical power splitter based on adiabatic tapered silicon waveguides," Opt. Lett. 41, 2053-2056 (2016).
20. D. González-Andrade, C. Lafforgue, E. Durán-Valdeiglesias, X. Le Roux, M. Berciano, E. Cassan, D. Marris-Morini, A. V. Velasco, P. Cheben, L. Vivien, and C. Alonso-Ramos, "Polarization- and wavelength-agnostic nanophotonic beam splitter," Sci. Rep. 9, 3604-3612 (2019).

21. J. Mu, P. T. Lin, L. Zhan, X. Duan, L. C. Kimerling, and A. M. Agarwal, "Silicon nitride $1 \times 8$ power splitter for mid-infrarred applications," in Laser Science 2014 (Optical Society of America, 2014), p. JW3A.26.

22. B. Sharma, K. Kishor, S. Sharma, and R. Makkar, "Design and simulation of broadband beam splitter on a silicon nitride platform for optical coherence tomography," Fiber Integrated Opt. 38, 247-257 (2019).

23. S. Guerber, C. Alonso-Ramos, D. Benedikovic, D. Pérez-Galacho, X. Le Roux, N. Vulliet, S. Crémer, L. Babaud, J. Planchot, D. Benoit, P. Chatraine, F. Leverd, D. Ristoiu, P. Grosse, D. Marris-Morini, L. Vivien, C. Baudot, and F. Boeuf, "Integrated SiN on SOI dual photonic devices for advance datacom solutions," in Proc. SPIE 10686, Silicon Photonics: From Fundamental Research to Manufacturing 2018, p. 106860W.

24. D. T. Spencer, D. Dai, Y. Tang, M. J. Heck, and J. E. Bowers, "Realization of a novel $1 \times \mathrm{N}$ power splitter with uniformly excited ports," IEEE Photon. Technol. Lett. 25, 36-39 (2012).

25.Z. Yuan and K. Wang, "Ultra broadband, low loss and polarization independent silicon nitrite integrated optical power splitter," in Asia Communications and Photonics (ACP) 2018 (IEEE, 2018), p. 1-3.

26. C. V. Poulton, M. J. Byrd, M. Raval, Z. Su, N. Li, E. Timurdogan, D. Coolbaugh, D. Vermeulen, and M. R. Watts, "Large-scale silicon nitride nanophotonic phased arrays at infrared and visible wavelengths," Opt. Lett. 42, 21-24 (2017).

27. S. Romero-García, F. Merget, F. Zhong, H. Finkelstein, and J. Witzens, "Silicon nitride CMOS-compatible platform for integrated photonics applications at visible wavelengths," Opt. Express 21, 14036-14046 (2013).

28. Lumerical Solutions, Inc., MODE Solutions (2019), http://lumerical .com.

29. S. Guerber, C. Alonso-Ramos, D. Benedikovic, E. Durán-Valdeiglesias, X. Le Roux, N. Vulliet, E. Cassan, D. Marris-Morini, C. Baudot, F. Boeuf, and L. Vivien, "Broadband polarization beam splitter on a silicon nitride platform for O-band operation," IEEE Photon. Technol. Lett. 30, 1679-1682 (2018).

30. R. Palmer, L. Alloatti, D. Korn, W. Heni, P. C. Schindler, J. Bolten, M. Kari, M. Waldow, T. Wahlbrink, W. Freude, C. Koos, and J. Leuthold, "Low-loss silicon strip-to-slot mode converters," IEEE Photonics J. 5, 2200409 (2013) 